\title{
Removing Shadows from Images
}

\author{
Zeinab Sadeghipour Kermani \\ School of Computing Science \\ Simon Fraser University \\ Burnaby, BC, V5A 1S6
}

\author{
Mark S. Drew \\ School of Computing Science \\ Simon Fraser University \\ Burnaby, BC, V5A 1S6
}

\begin{abstract}
We attempt to recover a $2 D$ chromaticity intrinsic variation of a single RGB image which is independent of lighting, without requiring any prior knowledge about the camera. The proposed algorithm aims to learn an invariant direction for projecting from $2 \mathrm{D}$ color space to the greyscale intrinsic image. We notice that along this direction, the entropy in the derived invariant image is minimized. The experiments conducted on various inputs indicate that this method achieves intrinsic $2 D$ chromaticity images which are free of shadows. In addition, we examined the idea to utilize projection pursuit instead of entropy minimization to find the desired direction.
\end{abstract}

\section{Introduction}

It has been investigated that many vision algorithms including image segmentation, object tracking or recognition may fail due to some specific illumination conditions [1][2][3]. To illustrate, shadows are the result of changes in lighting conditions in images which can cause some difficulties in the aforementioned set of algorithms. Hence, the problem of removing shadows from color images has stimulated increasing interest in the field of computer vision. One of the suggested solutions is based on the observation that greyscale intrinsic images extracted from the original color ones do not contain any shadows because they are not dependent on illumination conditions.

In [2], after generating a 2D chromaticity color map from the original image, they acquire a 1D illumination invariant image in which shadows are removed using the prior information about the camera. Afterwards, with the help of inverse conversions and shadow edges extracted from the source, they reconstruct a full color, shadow free image. This report is based on the method proposed in [3] that eliminates the need to calibrate camera. Instead, we seek the best direction for the projection line through minimizing entropy in the resulting greyscale image. Furthermore, the idea of using Independent Component Analysis (ICA) and projection pursuit to obtain the invariant direction is also discussed.

The rest of this report is organized as follows: in section 2, we study the details of the algorithm. Section 3 provides the results for a number of experiments and finally, the complete method is summerized in section 4 along with some suggestions for future work.

\section{System Model}

As stated in the previous section, our main goal in this project is to remove shadows from images through producing a greyscale intrinsic image. Note that our method assumed that camera sensors are fairly narrow-band or we applied spectral sharpening on them to simulate narrow-band behavior [4]. The light present in the images also should act similar to a Planckian light, such as daylight. In the following parts, we describe the steps of the algorithm in detail.

\subsection{Geometric Mean 2D Chromaticity Space}

Following the arguements and proofs in [3], at first we need to map the $3 \mathrm{D}$ original image to a $2 \mathrm{D}$ space. In order to accomplish this goal, we divided three color channels by the geometric mean $\sqrt[3]{R \times G \times B}$. Thus the chromaticity would be:

$$
c_{k}=R_{k} / \sqrt[3]{\prod_{i=1}^{3} R_{i}} \equiv R_{k} / R_{M}
$$

If we take the log of the above equation, we get:

$$
\rho_{k}=\log c_{k}=\log \left(\frac{s_{k}}{s_{M}}\right)+\frac{e_{k}-e_{M}}{T}, k=1 . .3,
$$

with

$$
\begin{gathered}
s_{k}=k_{1} \lambda_{k}^{-5} S\left(\lambda_{k}\right) q_{k}, s_{M}=\sqrt[3]{\prod_{j=1}^{3} s_{j}} \\
e_{k}=\frac{-k_{2}}{\lambda_{k}}, e_{M}=\frac{-k_{2}}{3 \sum_{j=1}^{p} \lambda_{j}},
\end{gathered}
$$



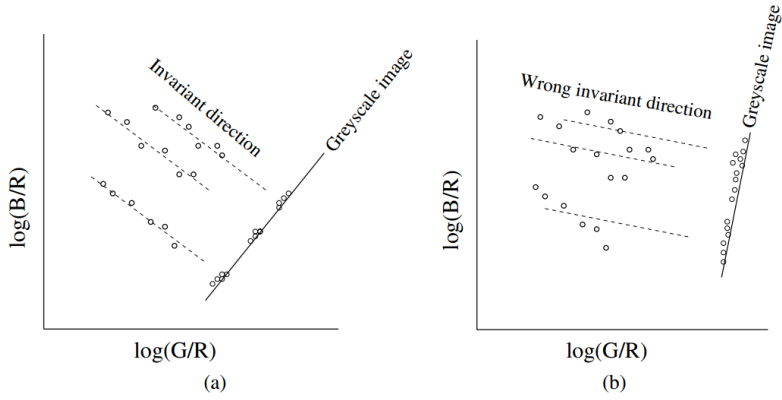

Figure 1: Inuition for using entropy minimization

However, we still have three components for the color space which should be projected into a proper plane to get a $2 \mathrm{D}$ representation of chromaticity. To accomplish this conversion, we form a plane $\left\{\chi_{1}, \chi_{2}\right\}$ such that straight lines in the color space $\rho$, each related to a single surface in the original image, remain straight in this plane[3]. As a result, if we plot 2D log-chromaticities, a pattern of parallel lines is identifieble that enables projection onto a line to derive an illuminant invariant image.

\subsection{Entropy Minimization}

To recover the greyscale intrinsic image, we require to learn the invariant direction in which the straight lines in the 2D chromaticity space mentioned above, point in. According to the observation in [3], the right invariant direction is the one that minimizes the entropy of the greyscale image. As illustrated in Fig. 1. (b), if we project onto a line which causes the entropy to be larger than the minimum, then the distribution of pixels along this line is not aggregated in a set of points. In other words, the desired line is perpendicular to the 2D log-chromaticity straight lines, leading to sharp peaks for corresponding surfaces after projection to $1 \mathrm{D}$, as in Fig. 1(a). Regarding the concept of entropy, these sharp peaks can be associated with small values for entropy.

To formulate this observation into algorithm steps, we require to iterate through every possible projection direction and select the one that minimizes the entropy. Accordingly, for angles $\theta=0^{\circ} . .180^{\circ}$, we produce the greyscale image $I$ from $2 \mathrm{D}$ chromaticity color map by:

$$
I=\chi_{1} \cos \theta+\chi_{2} \sin \theta,
$$

Next, for each angle, we compose histogram of the middle $90 \%$ of data with the intention to remove outliers. The bin width differes for every $\theta$ and is established using Scott's Rule [5]:

$$
\text { bin_width }=3.5 \operatorname{std}(I) N(I)^{1 / 3},
$$

and we calculate the entropy for each angle by

$$
\eta=-\sum_{i} p_{i} \log p_{i}
$$

with the height of the $i$-th bin denoted as $p_{i}$, to determine the minimum value.

\subsection{3-Vector Representation}

The final step of the method involves converting the greyscale intrinsic image back to a 3D representation for visualizing the results. To accomplish this, we form a $2 \times 2$ matrix $P_{\theta}$ as the projector for the candidate angle $\theta$ and utilize that to obtain 2 -vector $\chi_{\theta}$ as:

$$
\chi_{\theta}=P_{\theta} \chi
$$

Employing this 2D representation, we estimate the 3D color space and afterwards add a constant extra light to all pixels to retreive the original chromaticity [3].

\subsection{Algorithm Steps}

To sum up, we present the algorithm to reconstruct the greyscale intrinsic image in a number of explicit steps:

1. Project the original color image into a 2D logchromaticity space.

2. For $\theta=0^{\circ} . .180^{\circ}$

(a) Project the 2D representation of the image onto the line defined by the direction $\theta$ to retain a 1D greyscale image.

(b) Calculate the entropy for pixels in the resulting greyscale image.

(c) Select the angle that ensures the minimum entropy.

\subsection{Independent Component Analysis and Projection Pursuit}

The major purpose of the algorithm in [3] is to identify a line achieving a set of sharp peaks which are well-seperated from each other after pojection rather than attaining an outspread distribution for pixels. This concept can also be modeled by applying Independent Component Analysis (ICA) to the 2D log-chromaticity representation of the image.

The general definition for ICA concerns estimating a linear representation for the given mixed signal such that the components are statistically as independent as possible [6]. An alternative comprehension for ICA is maximizing the nongaussianity present in the data. Indeed this is one of the 
ICA estimation principles which states that each local maxima of nongaussianity in the given data with the constant variance, corresponds with one of the independent components. The preceding principle connects ICA with another method named projection pursuit which also has the objective to detect maximally nongaussian linear combinations of the data. In other words, the directions for projection pursuit are another interpretation for the independent components resulting from ICA.

Reconsidering the problem of verifying the correct invariant direction, we notice that recognizing the aforementioned set of peaks can be explained as maximizing the nongaussianity which is the intention of projection pursuit. Thus, a substitute for entropy minimization used in this project could be a robust implementation of ICA in [7] to learn the projection pursuit directions.

\section{Experiments and Results}

To evaluate our algorithm, we did three rounds of experiments. At first, we tried the algorithm for the data from [3] and extracted both the greyscale and $L_{1}$ chromaticity invariant images, shown in the last two columns of Fig. 2. As we can see, the method is quite successful in this round as the results are nearly shadow free.

The second time, we looked at the outputs of performing the approach on some JPEG images from unknown cameras which are provided in Fig. 3 (the dataset from [8]). We note that shadows are not removed as well as the previous case. It seems that a number of processing operations, e.g. compression in these images causes the procedure to fail since the assumptions made in the beginning of section 2 do not hold.

Finally, we investigate the idea of using projection pursuit instead of entropy minimization on the same set of images from Fig. 2 We used the first independet component of the 2D log-chromaticity to create the greyscale intrinsic images which are presented in the middle column of Fig. 4 The right column is repeated from Fig. 2 to compare two approaches. We notice that projection pursuit can work as effective as entropy minimizaion but is better in terms of performance; for instance, learning the invariant direction with entropy minimization takes almost 60 seconds for an original image of size $2048 \times 1152$ pixels while projection pursuit accomplishes the task in 10 seconds.

\section{Conclusions}

In this project, we implemented the algorithm demonstrated in [3] to reconstruct a greyscale and then an $L_{1}$ chromaticity illumination invariant image through entropy minimization with the aim to remove shadows from the original image. One of the crucial properties of suggested method is its independence on any specific information about camera or
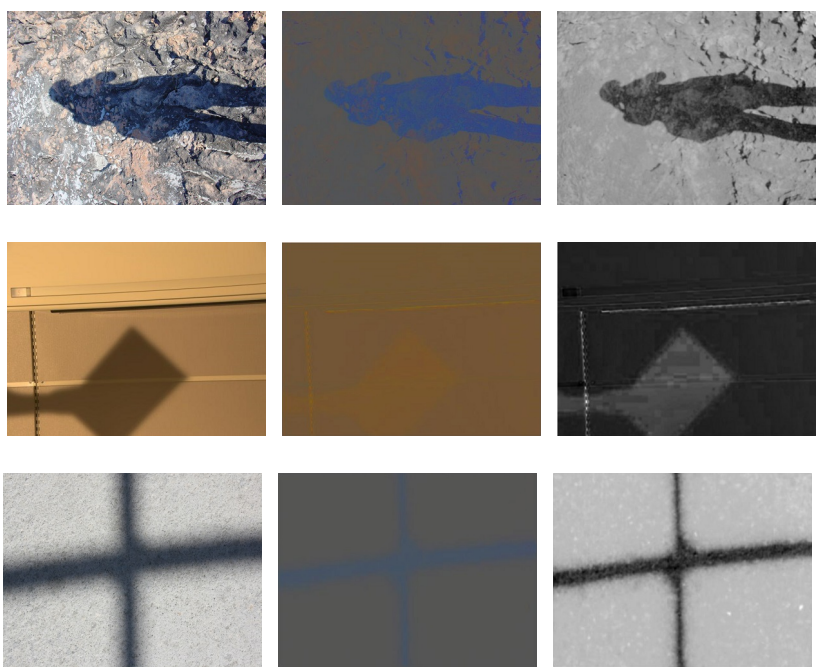

Figure 3: The result of applying entropy minimization method on JPEG images: columns show original image, $L_{1}$ chromaticity image and intrinsic greyscale image.
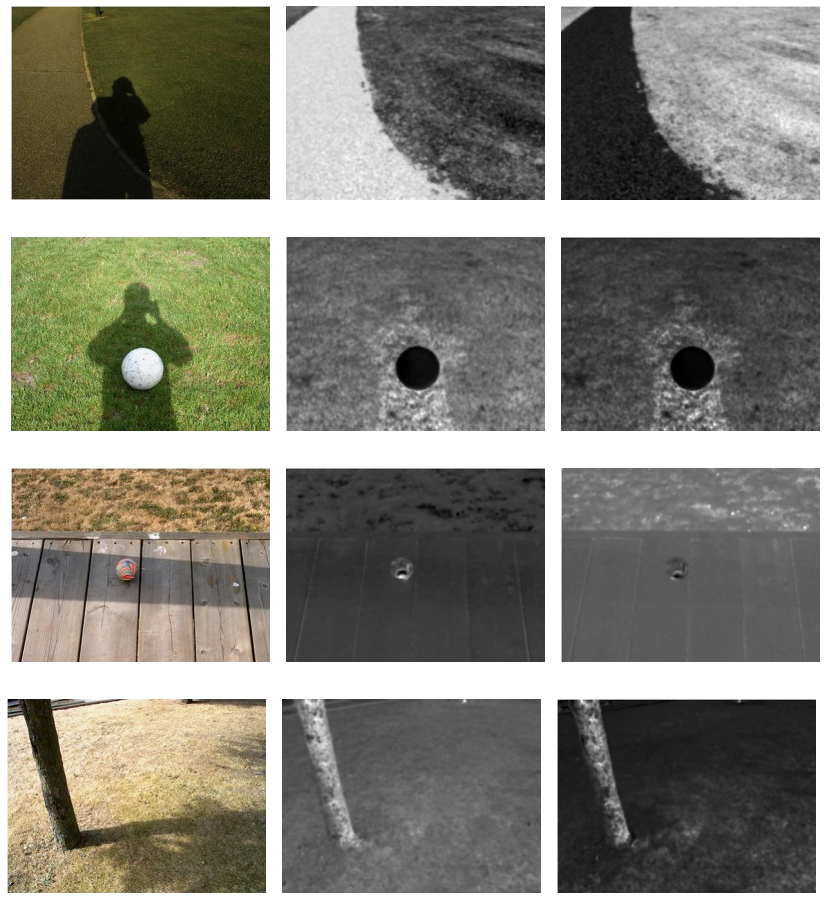

Figure 4: The result of applying projection pursuit method: columns show original image, intrinsic greyscale image obtained from projection pursuit and entropy minimization. 

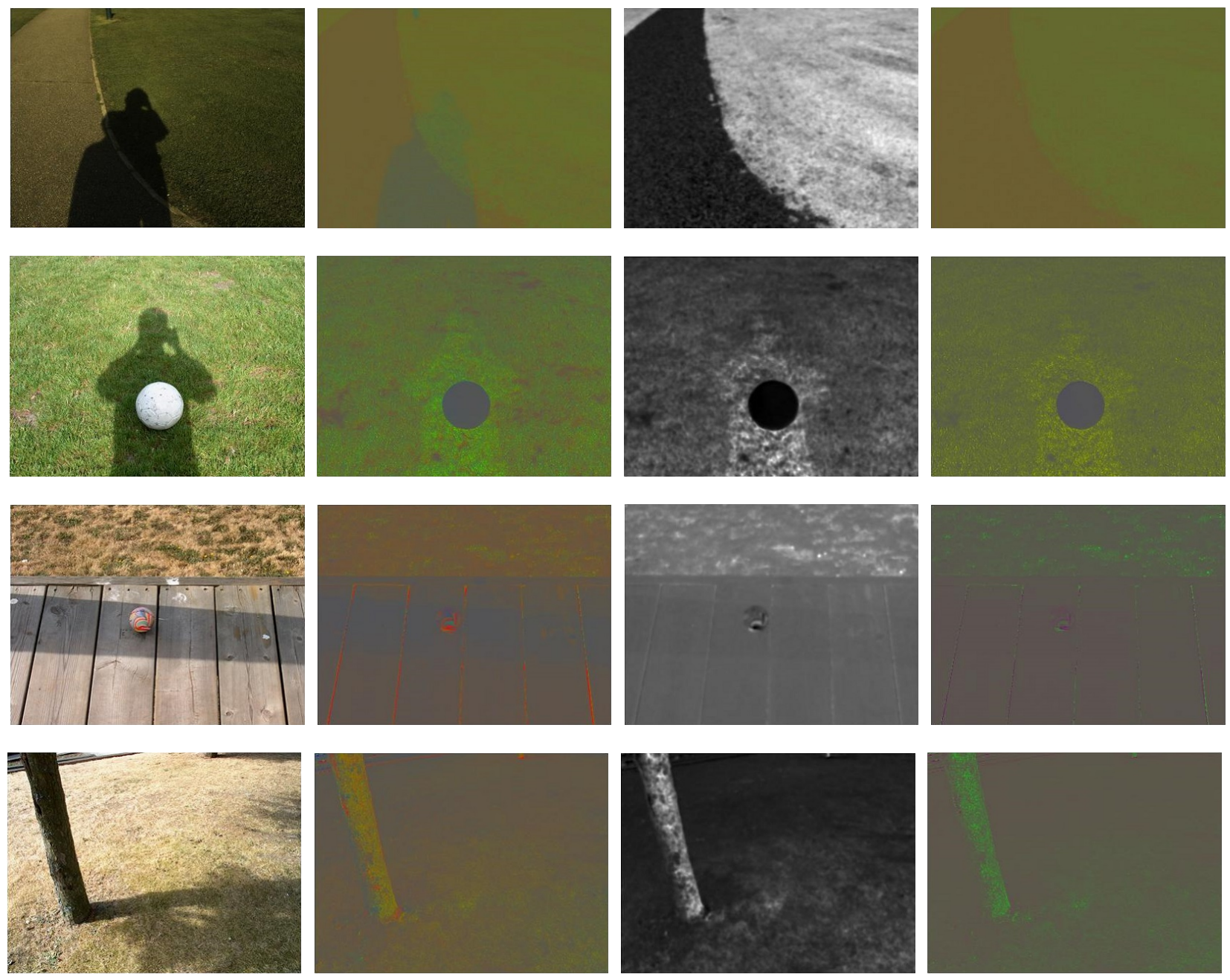

Figure 2: The result of applying entropy minimization method on data from [3]: columns show original image, $L_{1}$ chromaticity image, intrinsic greyscale image and invariant $L_{1}$ chromaticity. 
image. We also looked into projection pursuit and ICA to distinguish the invariant direction and came to this conclusion that it can be a replacement to entropy minimization.

Future work could concentrate on improving the approach to also work on JPEG images. Also it may be possible to enhance the reconstructed RGB shadow free image following the notion of projection pursuit.

\section{Acknowledgments}

I would like to show my gratitude to Ali Madooei for his helpful suggestions. I also thank Zhaopeng Cui for assistance to find an appropriate implementation for ICA.

\section{References}

[1] Graham D Finlayson and Mark S Drew, "4-sensor camera calibration for image representation invariant to shading, shadows, lighting, and specularities," in Computer Vision, 2001. ICCV 2001. Proceedings. Eighth IEEE International Conference on. IEEE, 2001, vol. 2, pp. 473-480.

[2] Graham D Finlayson, Steven D Hordley, and Mark S Drew, "Removing shadows from images," in Computer VisionECCV 2002, pp. 823-836. Springer, 2002.

[3] Graham D Finlayson, Mark S Drew, and Cheng Lu, "Intrinsic images by entropy minimization," in Computer Vision-ECCV 2004, pp. 582-595. Springer, 2004.

[4] Graham D Finlayson, Mark S Drew, and Brian V Funt, "Spectral sharpening: sensor transformations for improved color constancy," JOSA A, vol. 11, no. 5, pp. 1553-1563, 1994.

[5] David W Scott, Multivariate density estimation: theory, practice, and visualization, vol. 383, John Wiley \& Sons, 2009.

[6] Aapo Hyvärinen, Juha Karhunen, and Erkki Oja, Independent component analysis, vol. 46, John Wiley \& Sons, 2004.

[7] Vicente Zarzoso and Pierre Comon, "Robust independent component analysis by iterative maximization of the kurtosis contrast with algebraic optimal step size," Neural Networks, IEEE Transactions on, vol. 21, no. 2, pp. 248-261, 2010.

[8] Ruiqi Guo, Qieyun Dai, and Derek Hoiem, "Singleimage shadow detection and removal using paired regions," in Computer Vision and Pattern Recognition (CVPR), 2011 IEEE Conference on. IEEE, 2011, pp. 2033-2040. 